\title{
Sound Radiation Efficiency of a Baffled Rectangular Plate Excited by Harmonic Point Forces Using its Surface Resistance Matrix*
}

\author{
Jorge P. Arenas ${ }^{\dagger}$ \\ Institute of Acoustics, Universidad Austral de Chile, PO Box 567, Valdivia, Chile \\ Malcolm J. Crocker ${ }^{\dagger}$ \\ Department of Mechanical Engineering, Auburn University, AL 36849-5341, USA
}

(Received 29 May 2002; accepted 12 August 2002)

\begin{abstract}
In this paper the use of the acoustic radiation matrix is described. This matrix can be combined with the volume velocity vector on a discretised vibration surface to predict the total sound power radiated from the structure. In order to do so, the vibrating structure is simulated by a finite number of small piston sources flush-mounted on the surface. A definition of the acoustic radiation resistance matrix is presented and its main properties are discussed. The sound radiation from simply-supported and clamped-clamped plates was calculated numerically. Results are presented for the radiation efficiency of the natural modes of the plate when it is excited by a harmonic point force. The volume velocity distribution on the plate was calculated using a matrix approach. The vibration response of the clamped-clamped plate was determined using an approach based on the virtual work principle. In addition, experimental results for a real clamped aluminium plate are presented. The sound power radiated and the radiation efficiency were calculated from measurements of the normal velocity on the plate at discrete points without measuring the sound field. The sound power radiated from the plate and its radiation efficiency were measured directly using the classical sound intensity method, in order to test the accuracy of the resistance matrix approach. An expression to transform the velocities into vibration cross-spectra is presented in order to deal with the phase determination.
\end{abstract}

*This article was reviewed by referees unknown to either author

${ }^{\dagger}$ Member of the International Institute of Acoustics and Vibration (IIAV)

\section{INTRODUCTION}

The use of plates in aerospace structures is widespread. In recent years with the practical application of active sound and vibration control, several studies have been devoted to plate response subject to different kinds of excitation and with various boundary conditions. The vibration of a plate's surface and its interaction with the fluid medium results in the radiation of sound and a coupled vibro-acoustic problem. The solution for simply-supported plates is easy to obtain. It is much more difficult to obtain solutions for other boundary conditions. As computer resources become less expensive and more readily available, it is becoming increasingly practical to use computational methods to predict the propagation and radiation of sound and the behaviour of complex structural systems. An excellent review of the numerical techniques used to predict low frequency sound radiation has been presented by Atalla and Bernhard ${ }^{1}$. Usually, for low frequencies, the sound power is calculated using the data obtained from a finite mesh on the structural surface for which the vibration velocities have been computed. Subsequently, the sound power is computed using the boundary element method. In general the mesh needed to compute the sound radiation need not be as fine as the mesh needed to calculate the structural vibration. Therefore, sometimes acoustical analysis can be very inefficient. Fahnline ${ }^{2}$ has reported that the efficiency of acoustical analysis for subsonic structural waves is increased significantly by expanding the structural finite element mesh into a much coarser acoustical element mesh.

The sound power radiated by a plate can be calculated if its surface response is known., ${ }^{3,4}$ However, this makes it necessary to determine the acoustic field by using the Rayleigh integral (for the sound pressure distribution) and then to perform an integration over a closed control surface. Classical methods to find the surface response of a plate with complex boundary conditions include the superposition and Ritz methods using trial polynomials and trigonometric functions. ${ }^{5-7}$ These methods require the solution of simultaneous equations or high-order matrices, making the calculations of sound power radiated by vibrating structures even more difficult. An approach using the virtual work principle provides an easier methodology for calculating the surface response of a plate. ${ }^{8}$ A very good review concerning structural acoustics and the vibration behaviour of rectangular panels can be found in the work by Berry and Nicolas?.

Green functions can be theoretically derived for certain regular geometries. But for a complex and irregular geometric shape, or for a sound-radiating body not located in a free field, the function takes very complicated forms which are not easily determined mathematically. Since a homogeneous fluid at rest behaves like a linear elastic medium in response to small applied disturbances, the vibro-acoustic reciprocity principle can be used to avoid the Green function calculation. ${ }^{10}$ 